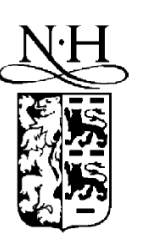

Journal logo

\title{
Degradation of High Resistivity Float Zone and Magnetic Czochralski N-type Silicon Detectors subjected to 2-MeV Electron Irradiation
}

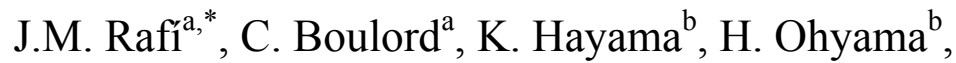

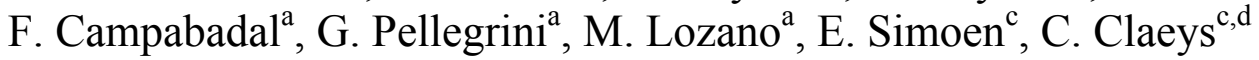 \\ a Instituto de Microelectrónica de Barcelona, IMB-CNM (CSIC), Campus UAB, 08193 Bellaterra, Spain \\ ${ }^{b}$ Kumamoto National College of Technology, 2569-2 Nishigoshi, Kumamoto 861-1102, Japan \\ ${ }^{c}$ IMEC, Kapeldreef 75, B-3001 Leuven, Belgium \\ ${ }^{d}$ KU Leuven, Electrical Engineering Department, Kasteelpark Arenberg 10, B-3001 Leuven, Belgium
}

Elsevier use only: Received date here; revised date here; accepted date here

\begin{abstract}
Particle tracking detectors made on high resistivity (HR) float zone (FZ) silicon are widely used in high energy physics experiments. It is known that the incorporation of oxygen in the FZ Si can lead to some improvement in the radiation hardness of the material. In this contribution we investigate the effects of $2 \mathrm{MeV}$ electron irradiation, up to a fluence of $5 \cdot 10^{16} \mathrm{e} / \mathrm{cm}^{2}$, on the electrical and carrier lifetime properties of p-on-n silicon diodes fabricated on different substrate materials, including HR standard and oxygenated FZ, as well as HR magnetic Czochralski silicon, with a higher intrinsic oxygen contents. A progressive degradation of the characteristics is observed for all devices, pointing to a generation of bulk damage. Interestingly, a significant increase of the effective carrier concentration is observed after the highest fluences for all materials. Under the limited experimental conditions studied, no significant changes are observed for diode characteristics subjected to a thermal annealing treatment at $80^{\circ} \mathrm{C}$. This degradation in the electrical properties should be taken into account for the use of such HR Si materials under high energy electron environments. C) 2008 Elsevier Science. All rights reserved
\end{abstract}

Keywords: High resistivity silicon; Electron irradiation; Magnetic Czochralski; Float Zone; Diffusion oxygenated FZ; Carrier lifetime

\section{Introduction}

Particle tracking detectors made on high resistivity (HR) float zone (FZ) silicon are widely used in high energy physics experiments. Results from the CERN
RD48 [1] and RD50 [2] collaborations have shown that diffusion oxygenated FZ (DOFZ) silicon can better withstand the high hadron fluences expected for 10 years operation of the Large Hadron Collider at CERN [1]. It is known that electron irradiation

\footnotetext{
* Corresponding author. Tel.: +34 93 5947700; fax: +34 93 5801496; e-mail: jmrafi@cnm.es.
} 
may also introduce significant bulk damage in silicon devices and this is of particular interest for certain environments like space applications or linear colliders. Type inversion after electron irradiation was firstly observed in p-type Czochralski (CZ) Si solar cells for space applications [3]. More recently, n-type to p-type conversion was also encountered after irradiation with ${ }^{60} \mathrm{Co} \gamma$ rays [4], which can produce low-energy electrons by Compton effect. Such type conversion after $\gamma$-rays was not observed in oxygen-rich DOFZ material [4]. The impact of highenergy electron irradiation on HR silicon for detector applications has been assessed in a series of recent publications [5-7], revealing the appearance of bulktype inversion in either standard or oxygenated HR FZ, with no clear advantage in using DOFZ material.

Recently, new semiconductor industry interests and developments have enabled the production of magnetic Czochralski (MCZ) Si wafers with sufficiently $\mathrm{HR}$ and with a well-controlled high concentration of interstitial oxygen $\left(\left[\mathrm{O}_{\mathrm{i}}\right]\right)$. Although there are already some first published studies assessing the radiation hardness of detectors fabricated on the new HR MCZ material, these have mostly concentrated on neutron, proton and $\gamma$ irradiations $[8,9]$. The impact of electron irradiation on the new HR MCZ material remains still unclear as no type inversion has been observed and the first results point to either an effective dopant concentration lowering with $900 \mathrm{MeV}$ e-irradiation [10] or just the opposite trend with $\gamma$-rays [9].

In order to shed some further light on the behavior of the HR MCZ n-type material under electron irradiation, silicon diodes including standard and oxygenated HR FZ as control materials were subjected to $2 \mathrm{MeV}$ e-irradiations.

\section{Experimental}

The p-on-n diodes under study were manufactured at IMB-CNM following a well-established fabrication process $[11,12]$. The starting material included HR FZ, DOFZ and MCZ Si wafers (Table 1). $2 \mathrm{MeV}$ e-irradiations were performed at room temperature for different fluences $(\phi)$ between $5 \cdot 10^{11}$ and $5 \cdot 10^{16} \mathrm{e} / \mathrm{cm}^{2}$ using the Dynamitron facility at Takasaki-JAERI, in Japan.
Table 1. Main specifications of the three HR Si substrates studied.

\begin{tabular}{cccc}
\hline Substrate & HR MCZ & HR FZ & HR DOFZ \\
\hline Supplier & Okmetic & Topsil & Topsil \\
Type & $n$ & $n$ & $n$ \\
Orientation & $<100>$ & $<100>$ & $<100>$ \\
$\begin{array}{c}\text { Thickness }(\mathrm{d}) \\
{[\mu \mathrm{m}]}\end{array}$ & $300 \pm 10$ & $280 \pm 15$ & $280 \pm 15$ \\
$\begin{array}{c}\text { Resistivity } \\
{[\mathrm{k} \Omega \cdot \mathrm{cm}]}\end{array}$ & $1.0 \pm 0.1$ & $4.5 \pm 0.6$ & $2.5 \pm 0.1$ \\
$\begin{array}{c}\text { Dopant conc. } \\
{\left[\mathrm{x} 10^{12} \mathrm{~cm}^{-3}\right]}\end{array}$ & $3.9 \pm 0.4$ & $0.9 \pm 0.1$ & $1.6 \pm 0.1$ \\
$\begin{array}{c}\text { Average }\left[\mathrm{O}_{\mathrm{i}}\right] \\
{\left[\mathrm{x} 10^{17} \mathrm{~cm}^{-3}\right]}\end{array}$ & $4.6 \pm 0.2$ & $<0.09$ & $1.7 \pm 0.4$ \\
\hline
\end{tabular}

Before and after irradiation, the electrical performance was evaluated by measuring the current-voltage $\left(\mathrm{I}_{\text {leak }}-\mathrm{V}\right)$ and capacitance-voltage $(\mathrm{C}$ V) characteristics. Exploratory minority carrier recombination lifetime $\left(\tau_{\mathrm{r}}\right)$ measurements were performed by means of a microwave photoconductance ( $\mu \mathrm{W}$-PCD) Semilab WT1000 setup [13].

\section{Discussion}

Figure 1 shows typical radiation-induced degradation of leakage current density $\left(\mathrm{J}_{\text {leak }}=\mathrm{I}_{\text {leak }} /\right.$ Area $)$ for $\mathrm{HR} \mathrm{MCZ}$ detectors, similar results were obtained for their HR FZ and HR DOFZ counterparts. This points to a significant increase in the density of generation-recombination centers and bulk damage under electron irradiation. Interestingly, a significant degradation of capacitance versus voltage curves was also observed after the highest irradiation fluences (Fig. 2), showing an increase of the reverse bias voltage required to achieve full wafer thickness depletion $\left(\mathrm{V}_{\mathrm{FD}}\right)$ [11], what is associated with an effective carrier concentration $\left(\mathrm{N}_{\text {eff }}\right)$ increase after the highest fluences (Figs. 3 and 4).

Regarding the leakage current increase (Fig. 1), as expected, a similar trend is observed for all the different materials $\left(\mathrm{I}_{\mathrm{vol}}=\alpha \cdot \phi\right.$, with $\mathrm{I}_{\mathrm{vol}} \equiv \mathrm{J}_{\text {leak }} /$ depth, with $\alpha$ values around $5.5 \cdot 10^{-20} \mathrm{~A} / \mathrm{cm}$ ) (Fig. 5), in good agreement with those published in the literature for lower resistivity (standard) Si subjected to similar energy e-irradiations [14]. From $\alpha$, an experimental estimation for the relative hardness factor of $2 \mathrm{MeV}$ electrons with respect to $1 \mathrm{MeV}$ neutrons gives a value of $\sim 6.9 \cdot 10^{-4}$, that is significantly smaller than the estimated value $\left(2.49 \cdot 10^{-2}\right)$ obtained from the 


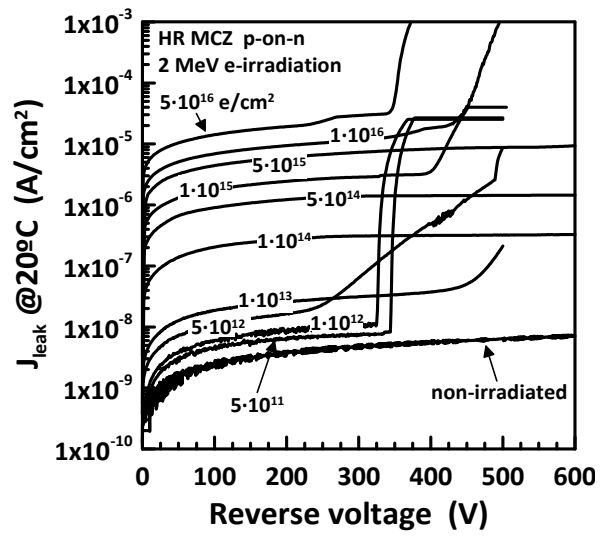

Fig. 1. $\mathrm{J}_{\text {leak }}$ versus voltage for irradiated HR MCZ detectors.

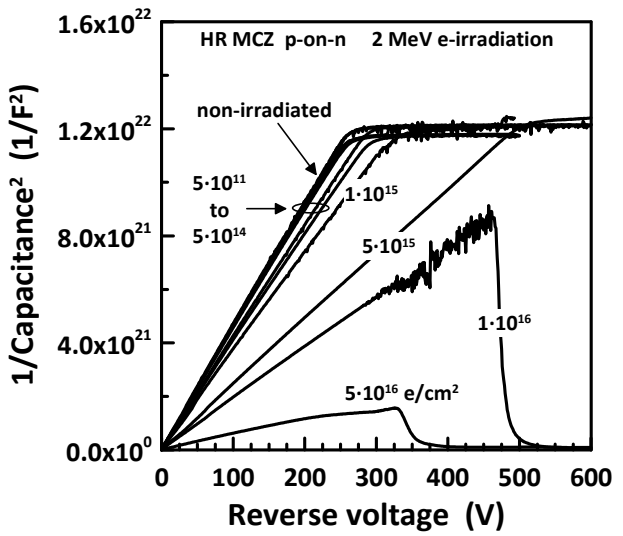

Fig. 2. $1 / \mathrm{C}^{2}$ versus reverse voltage for Fig. 1 detectors.

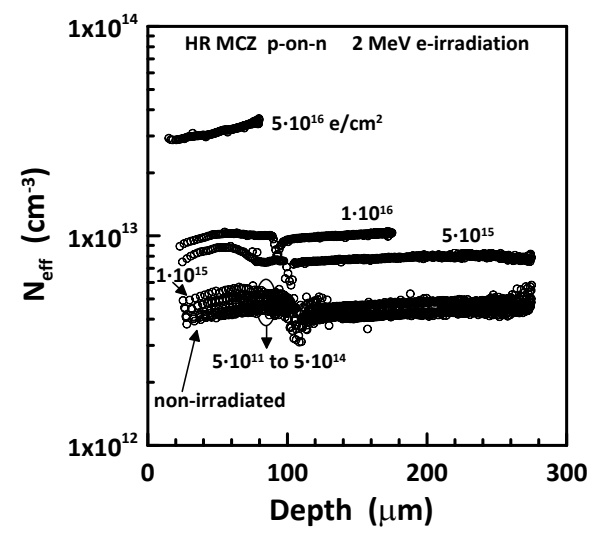

Fig. 3. Effective doping versus depth for Fig. 2 detectors.

NIEL ratio [14,15]. The deviation from the NIEL scaling can be explained by a more pronounced introduction of point defects with respect to cluster formation under such energy e-irradiation $[14,15]$.

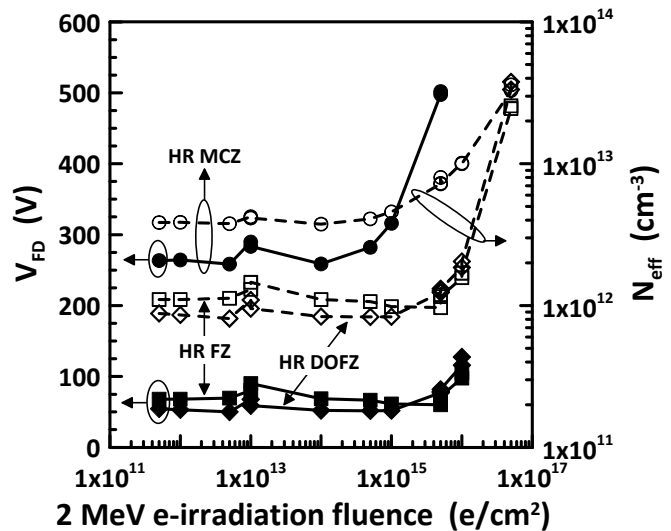

Fig. 4. $\mathrm{V}_{\mathrm{FD}}$ and $\mathrm{N}_{\text {eff }}$ versus e-irradiation fluence for HR MCZ, HR FZ and HR DOFZ detectors.

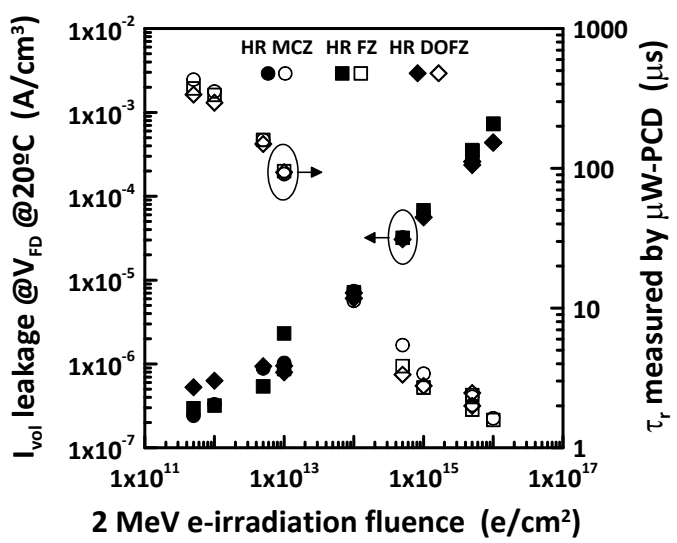

Fig. 5. Leakage current density $\left(I_{\text {vol }}=J_{\text {leak }} /\right.$ depth $)$ and $\tau_{\mathrm{r}}$ measured by $\mu \mathrm{W}-\mathrm{PCD}$ as a function of e-irradiation fluence.

Interestingly, the obtained $\mu \mathrm{W}-\mathrm{PCD} \tau_{\mathrm{r}}$ values monotonously decrease with e-irradiation fluence (Fig. 5) and a certain correlation is found with the degradation of the generation lifetime $\left(\tau_{\mathrm{g}}\right)$ extracted from $J_{\text {leak }}$ versus depth plots (Figs. 6 and 7) [16]. By assuming a $\tau_{\mathrm{g}} / \tau_{\mathrm{r}}$ ratio around 10 for low e-fluences $(\leq$ $\left.1 \cdot 10^{15} \mathrm{e} / \mathrm{cm}^{2}\right)$, a dominant effective trap level situated at about $60 \mathrm{mV}$ from the intrinsic midgap position could even be estimated.

Finally, in order to evaluate the thermal stability of the radiation-induced damage, a few irradiated diodes were subjected to thermal annealing treatments at $80^{\circ} \mathrm{C}$. Under the limited experimental conditions studied, no significant changes have been observed for the measured $\mathrm{I}-\mathrm{V}$ and $\mathrm{C}-\mathrm{V}$ characteristics (Figs. 8 and 9), as well as for the generation and recombination lifetimes. 


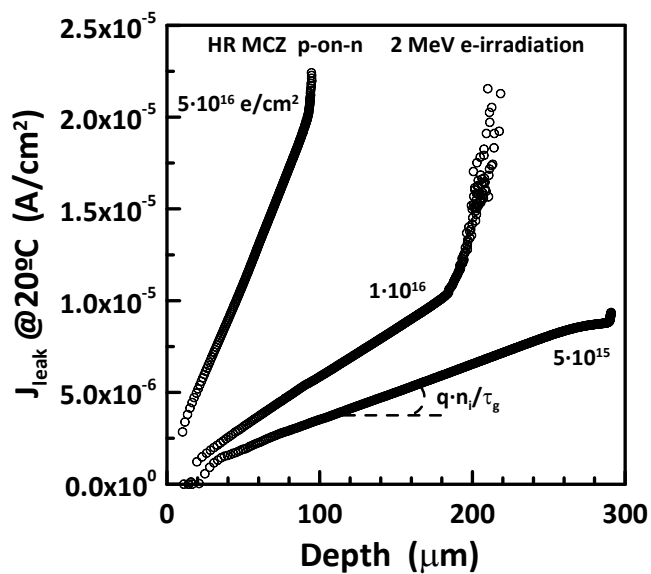

Fig. 6. $\mathrm{J}_{\text {leak }}$ versus depth for three irradiated HR MCZ detectors. $\tau_{\mathrm{g}}$ is extracted from the slope of the linear region [16].

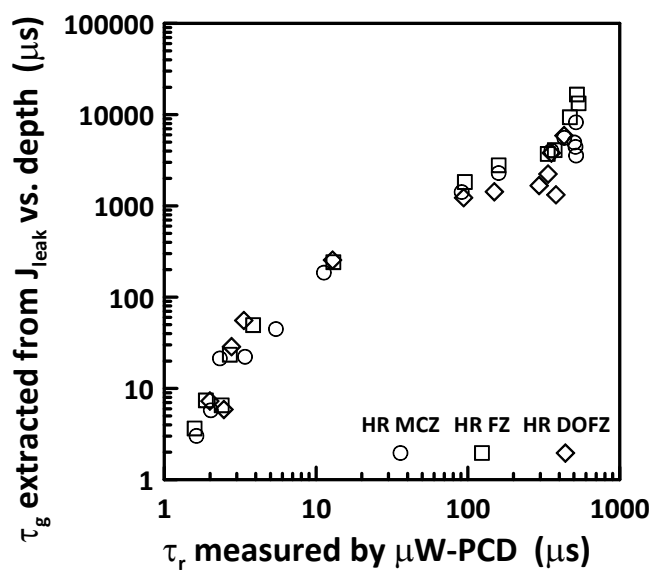

Fig. 7. $\tau_{\mathrm{g}}$ versus $\tau_{\mathrm{r}}$ for various irradiated $\&$ non-irradiated devices.

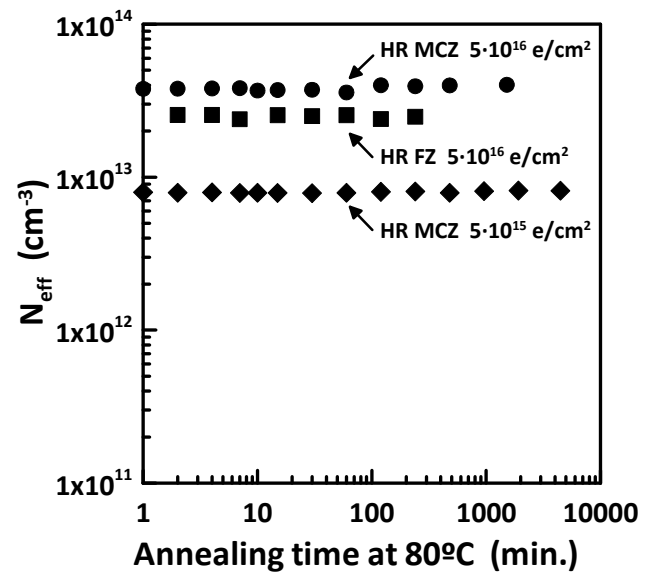

Fig. 8. $\mathrm{N}_{\text {eff }}$ as a function of annealing time at $80^{\circ} \mathrm{C}$ for three different irradiated detectors.

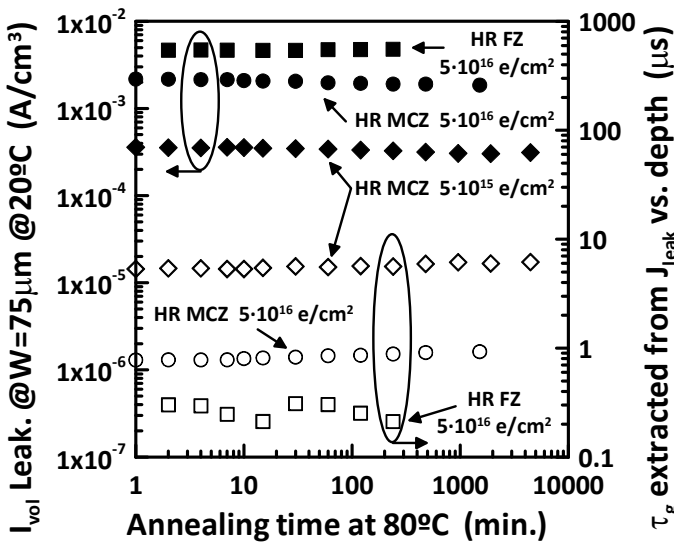

Fig. 9. Leakage current density $\left(\mathrm{I}_{\mathrm{vol}}=\mathrm{J}_{\text {leak }} /(@\right.$ depth $\left.=75 \mu \mathrm{m})\right)$ and $\tau_{\mathrm{g}}$ as a function of annealing time at $80^{\circ} \mathrm{C}$ for Fig. 8 devices.

\section{Conclusions}

A progressive degradation of the $\mathrm{I}-\mathrm{V}$ curves is observed for all HR substrate diodes subjected to 2 $\mathrm{MeV}$ e-irradiation. Interestingly, a significant increase of the effective carrier concentration is also observed after the highest fluences for all materials. Under the experimental conditions studied, no impact of a thermal annealing treatment at $80^{\circ} \mathrm{C}$ has been appreciated. This degradation in the electrical properties should be considered for the use of such HR Si materials under high energy e- environments.

\section{Acknowledgments}

The Ramón y Cajal program of The Spanish Ministry of Science and Innovation is acknowledged.

\section{References}

[1] G. Lindström, et al., Nucl. Instr. \& Meth. A 466 (2001), 308.

[2] http://rd50.web.cern.ch/rd50

[3] S.J. Taylor, et al., Appl. Phys. Lett.70 (1997), 2165.

[4] B. Dezillie, et al., IEEE Trans. Nucl. Sci. 47 (2000), 1892.

[5] I. Rachevskaia, et al., Nucl. Instr. \& Meth. A 485 (2002), 126.

[6] S. Dittongo, et al., Nucl. Instr. \& Meth. A 512 (2003), 77.

[7] S. Dittongo, et al., Nucl. Instr. \& Meth. A 530 (2004), 110.

[8] E. Touminen, et al., IEEE Trans. Nucl. Sci. 50 (2003), 1942.

[9] Z. Li, et al., IEEE Trans. Nucl. Sci. 51 (2004), 1901.

[10] S. Dittongo, et al., Nucl. Instr. \& Meth. A 546 (2005), 300.

[11] C. Martínez, et al., IEEE Trans. Nucl. Sci. 49 (2002), 1377.

[12] G. Pellegrini, et al., Nucl. Instr. \& Meth. A 548 (2005), 355.

[13] http://www.semilab.hu

[14] A. Vasilescu and G. Lindstroem, On-line compilation: http://sesam.desy.de/members/gunnar/Si-dfuncs.html

[15] G.P. Summers, et al., IEEE Trans. Nucl. Sci. 40 (1993), 1372.

[16] Y. Murakami, et al., J Appl. Phys. 75 (1994), 3548. 